Dynamic Optimal Scheduling Method and Its Application for Converter Fault in Steelmaking and Continuous Casting Production Process

Yu, Shengping and Chai, Tianyou and Wang, Hong and Pang, Xinfu and Zheng, Binglin

2011

MIMS EPrint: 2011.33

Manchester Institute for Mathematical Sciences

School of Mathematics

The University of Manchester

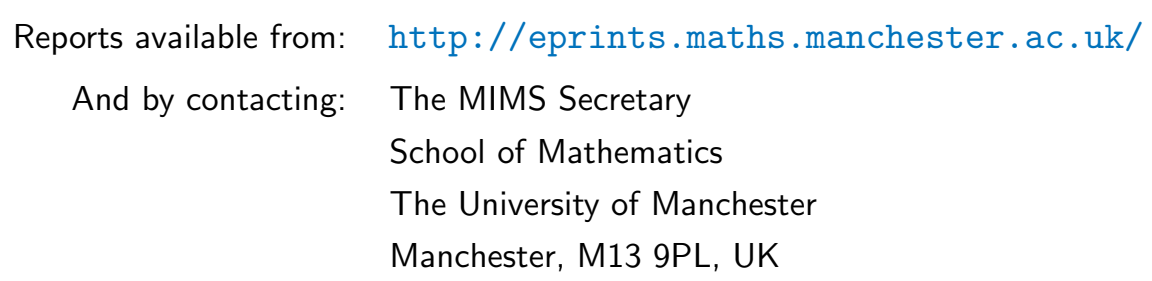

ISSN 1749-9097 


\title{
Dynamic Optimal Scheduling Method and Its Application for Converter Fault in Steelmaking and Continuous Casting Production Process
}

\author{
Shengping Yu*, Tianyou Chai ${ }^{* * *}$, Hong Wang ${ }^{* * * *}$, Xinfu Pang*, Binglin Zheng* \\ * State Key Laboratory of Integrated Automation for Process Industry (Northeastern University), \\ Shenyang, China,110004 (Tel: +86-24-83689665-8013; e-mail: spyu@mail.neu.edu.cn) \\ ** National Metallurgical Automation Engineering Technology Research Center, Northeastern University, \\ Shenyang, China, 110004 (e-mail: tychai@mail.neu.edu.cn) \\ *** Control Systems Centre, School of Electrical and Electronic Engineering, the University of Manchester, \\ $P O$ Box 88,Manchester M60 1QD, UK
}

\begin{abstract}
In steelmaking and continuous casting (SMCC) production process, converter fault can lead to unexpected changes to the pre-specified converter-continuous caster production mode so that the original scheduling plan becomes unrealizable. In this paper, the dynamic scheduling problem in response to converter fault is firstly analyzed. This is then followed by the establishment of a novel multi-objective nonlinear programming model (MONPM) by introducing the production mode parameter $\alpha$, production schedule parameter $\beta$ and $\chi$. The proposed method considers changes in production mode, production schedule of charge, the interval characteristics of processing time. In specific, a two-stage dynamic optimal scheduling method is proposed including the production path planning of charges (PPP) and the production time scheduling (PTS). As a result, a dynamic optimal scheduling software system (DOSSS) is developed and is successfully applied to the scheduling of the largest iron and steel company (BaoSteel) in China. The real-time application shows that the proposed method can efficiently reduce scheduling time, significantly increase the outputs of converters and dramatically shorten the redundant waiting time for molten steel.
\end{abstract}

Keywords: steelmaking and continuous casting; dynamic optimization; production mode; nonlinear programming; scheduling system.

\section{INTRODUCTION}

SMCC production process is the core process in modern iron and steel making ${ }^{[1]}$. There are high expectations for molten steel temperature, molten steel composition, processing time and transportation time to be optimized. Ultimately it is expected that the production capacity is maximized for a continuous production. Various disturbances often occur and examples of the disturbances are delayed starting time, failure temperature of molten steel, unqualified compositions of molten steel and machine failures etc due to variations of raw material composition, production operating condition and equipment condition. A rapid and effective dynamic scheduling is therefore important.

In this context, much effort has been made on the research into the schedule for SMCC. The auction-based method is used to solve the schedule problem of SMCC (Kumar, et al. [2006]). Using 'just-in-time (JIT)' principle, in (Tang, et al. [2002]) the nonlinear integral programming model is established for solving machine conflicts after the charge was

\footnotetext{
^ This work is jointly supported by the Chinese National Fundamental Research Program (No. 2009CB320601), the National Natural Science of China (No. 60534010, 60828007), the Funds for Creative Research Groups of China (No. 60821063), and 111 Project (No. B08015), UK EPSRC (EP/E050441/1), the Natural Science Foundation of Nigbo City (2010A610135) and the Innovation and Entrepreneurship Fund of Ningbo City (2010B710019).
}

scheduled, and it is then converted into a linear programming model which can be solved using standard linear programming. In (Tang, et al. [2002]; Xuan, et al. [2007]), SMCC procedure is abstracted into a three-stage hybrid flow shop problem, and static schedule model is established which can be solved using the Lagrangian relaxation method. In (Harjunkoski, et al. [2001]), a decomposition scheme is proposed that generates smaller programs which can often be solved to obtain global optimality. In (Cowling, et al. [2003]; Mohanty, et al. [2004]; Ouelhadj, et al. [2004]), a multi-agent technology is used to study the dynamic scheduling. Moreover, in (Tang, et al. [2010]) the coordinated scheduling problem between production and transportation in a steelmaking shop is explored. A beam search algorithm is used to solve schedule problem for SMCC (Pacciarelli, et al. [2004]). A feedback control method is studied for real-time schedule problem ( $\mathrm{Lu}$, et al. [2002]). However, the existing literatures are mainly focused on the static scheduling problem, and in particular these methods do not consider the scheduling when converters are subjected to failure which changes the production mode. In addition, the widely used manual scheduling method responses slowly and makes the scheduling results not optimal. In this paper, a novel multiobjective nonlinear programming model is established by introducing the production mode parameter $\alpha$, production schedule parameter $\beta$ and $\chi$. In specific, a two-stage dynamic optimal scheduling method is proposed including the PPP 
and the PTS. As a result, a DOSSS is developed and is successfully applied to the scheduling of the largest iron and steel company (BaoSteel) in China. The real-time application shows that the proposed method can efficiently reduce scheduling time, significantly increase the outputs of converters and dramatically shorten the redundant waiting time for molten steel.

\section{DYNAMIC SCHEDULING FOR SMCC PRODUCTION PROCESS}

\subsection{SMCC Production Process}

Taking BaoSteel plant for example, one production mode is shown in Fig. 1. At steel making stage, molten irons of high temperature are poured into a number of converters to remove harmful gases and residuals. Moreover, certain percent of scrapped iron are added to adjust the component of molten steel to meet the product specifications. Molten steel then undergoes refining processes to produce molten steel of the correct chemical components and temperature. At the final continuous casting stage, molten steel is drained into a tundish in continuous caster and flows into riming machine of continuous caster to be cooled into the required slabs.

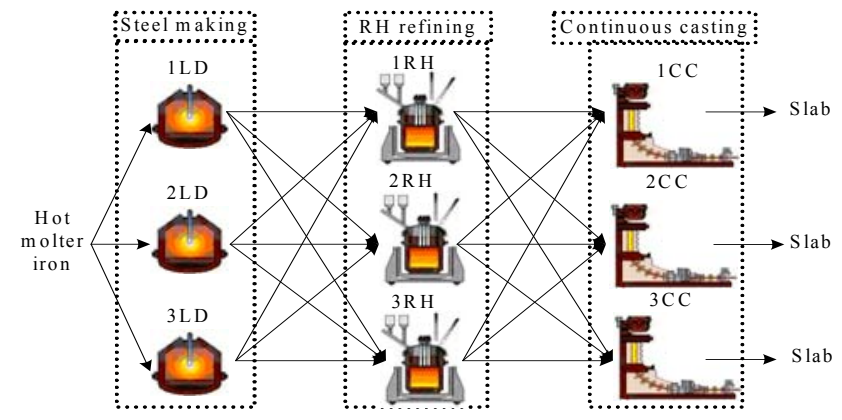

Fig. 1. SMCC production process

This process can therefore be classified into three groups, namely the converter group, $\mathrm{RH}$ refining group and continuous caster group. These three groups connected with each other in series. In this paper, we denote $M_{i j}$ as the the $i$ th machine in the $j$ th group $(j=1,2,3)$. This means that $j=1$ indicates converter group, $j=2$ stands for $\mathrm{RH}$ group and $j=3$ represents the continuous caster group.

\subsection{Dynamic Scheduling for the SMCC Production Process}

Ladle is used to carry molten steel on the processing of all processes, and the transportation of ladle among the processes is called a Charge. All charges which are continuously drained into the tundish in one continuous caster are called a Cast. For the system considered in this paper (see Fig. 1) we denote $L_{k q}$ for the $k$ th charge of the $q$ th cast, and $q=1,2,3, k=1, \cdots, n_{q}$, where $n_{q}$ indicates the total charge number of the $q$ th cast.

When a machine either breaks down or needs to be repaired, the initial production tasks should be transferred to another parallel machine of that group. In this case the production mode is different from the original scheduling condition. Therefore, the purpose of scheduling is to arrange an optimal production path and a set of processing units for a given charge so that any charge can be continuously processed in the casters, the redundant waiting time is minimized and the starting time of each cast is equal to the specified time.

\subsection{Dynamic Scheduling Objectives for SMCC Production Process}

Assuming that the current processing is for the $k$ th charge of the $q$ th cast, then we define $x_{i j}(k, q)$ as its starting time on the $i$ th machine in the $j$ th group, and $y_{i j}(k, q)$ as the corresponding processing time.

Then in the dynamic scheduling, it is expected for the caster that $x_{i 3}(1, q)$ is made as close as possible to its ideal starting time $x_{i 3}^{*}(1, q)$. This means that the following performance functions need to be minimized.

$$
\begin{aligned}
& J_{1}=\left(1-\beta_{3}^{1,1}\right)\left|x_{1,3}(1,1)-x_{1,3}^{*}(1,1)\right| \\
& J_{2}=\left(1-\beta_{3}^{1,2}\right)\left|x_{2,3}(1,2)-x_{2,3}^{*}(1,2)\right| \\
& J_{3}=\left(1-\beta_{3}^{1,3}\right)\left|x_{3,3}(1,3)-x_{2,3}^{*}(1,3)\right|
\end{aligned}
$$

where $\beta_{j}^{k q}$ represents the processing state of the $k$ th charge of the $q$ th cast of the $j$ th group. It is set to ' 1 ' if the charge is being processed. Otherwise it is set to ' 0 '.

It is expected that $x_{i 3}(k+1, q)$ is made as close as possible to the end time $x_{i 3}(k, q)+y_{i 3}(k, q)$. For this purpose, the following performance functions need to be minimized.

$$
\begin{aligned}
& J_{4}=\left(1-\beta_{3}^{k+1,1}\right)\left(x_{1,3}(k+1,1)-\left(x_{1,3}(k, 1)+y_{1,3}(k, 1)\right)\right) \quad k=1, \cdots, n_{1}-1 \\
& J_{5}=\left(1-\beta_{3}^{k+1,2}\right)\left(x_{2,3}(k+1,2)-\left(x_{2,3}(k, 2)+y_{2,3}(k, 2)\right)\right) \quad k=1, \cdots, n_{2}-1 \\
& J_{6}=\left(1-\beta_{3}^{k+1,3}\right)\left(x_{3,3}(k+1,3)-\left(x_{2,3}(k, 3)+y_{2,3}(k, 3)\right)\right) \quad k=1, \cdots, n_{3}-1
\end{aligned}
$$

We define $\omega_{p r}^{i j}$ as the transportation time from the $i$ th machine of $j$ th group to $p$ th machine of $r$ th group. Then it is expected that the redundant waiting time is minimized. This means that the following performance functions need to be minimized.

$$
\begin{gathered}
J_{7}=\left(1-\beta_{2}^{k q}\right)\left(x_{i, 2}(k, q)-\omega_{p, 1}^{i, 2}-\left(x_{p, 1}(k, q)+x_{p, 1}(k, q)\right)\right) \\
q=1,2,3, k=1, \cdots, n_{q}, i=1,2,3, p=1,2,3 \\
J_{8}=\left(1-\beta_{3}^{k q}\right)\left(x_{i, 3}(k, q)-\omega_{p, 2}^{i, 3}-\left(x_{p, 2}(k, q)+x_{p, 2}(k, q)\right)\right) \\
q=1,2,3, k=1, \cdots, n_{q}, i=1,2,3, p=1,2,3
\end{gathered}
$$

The $S_{i j}^{k q}$ is defined as the immediate successor charge of the $k$ th charge of the $q$ th cast on the $i$ th machine in the $j$ th group.

It is expected that $x_{i, 1}\left(S_{i, 1}^{k q}, h\right)$ is made as close as possible to the end time $x_{i I}(k, q)+y_{i l}(k, q)$. This means that the following performance functions need to be minimized.

$$
\begin{gathered}
J_{9}=(1-\alpha)\left(1-\beta_{1}^{S_{1,1}^{k,}, h}\right)\left(x_{1,1}\left(S_{1,1}^{k q}, h\right)-x_{1,1}(k, q)-x_{1,1}(k, q)\right) \\
q=1,2,3, k=1, \cdots, n_{q}
\end{gathered}
$$




$$
\begin{gathered}
J_{10}=(1-\alpha)\left(1-\beta_{1}^{S_{2,1}^{k q}, h}\right)\left(x_{2,1}\left(S_{2,1}^{k q}, h\right)-x_{2,1}(k, q)-x_{2,1}(k, q)\right) \\
q=1,2,3, k=1, \cdots, n_{q} \\
J_{11}=(1-\alpha)\left(1-\beta_{1}^{S_{3,}^{k q}, h}\right)\left(x_{3,1}\left(S_{3,1}^{k q}, h\right)-x_{3,1}(k, q)-x_{3,1}(k, q)\right) \\
q=1,2,3, k=1, \cdots, n_{q}
\end{gathered}
$$

where $\alpha$ indicates the production mode parameter. It is set to ' 1 ' if production mode is three converter-three continuous caster. Otherwise it is set to ' 0 '.

It is expected that each cast is quickly finished as far as possible. The following performance functions need to be minimized. $T^{*}$ represents the production schedule time.

$$
\begin{aligned}
& J_{12}=x_{1,3}\left(n_{1}, 1\right)+y_{1,3}\left(n_{1}, 1\right)-T^{*} \\
& J_{13}=x_{2,3}\left(n_{2}, 2\right)+y_{2,3}\left(n_{2}, 2\right)-T^{*} \\
& J_{14}=x_{3,3}\left(n_{3}, 3\right)+y_{3,3}\left(n_{3}, 3\right)-T^{*}
\end{aligned}
$$

\subsection{Dynamic Scheduling Constraints for SMCC Production Process}

In the SMCC production, it must have been processed on the former unit before it can be processed on the next one for each charge. Therefore the following constraints need to be met.

$$
\begin{gathered}
\left(1-\beta_{2}^{k q}\right)\left(x_{i, 2}(k, q)-\omega_{p, 1}^{i, 2}-\left(x_{p, 1}(k, q)+y_{p, 1}(k, q)\right)\right) \geq 0 \\
q=1,2,3, k=1, \cdots, n_{q}, i=1,2,3, p=1,2,3 \\
x_{i, 3}(k, q)-\omega_{p, 2}^{i, 3}-\left(x_{p, 2}(k, q)+x_{p, 2}(k, q)\right) \geq 0 \\
q=1,2,3, k=1, \cdots, n_{q}, i=1,2,3, p=1,2,3
\end{gathered}
$$

The adjacent charges in the same machine must be processed one by one, which is represented by the following constraints.

$$
\begin{gathered}
\left(1-\beta_{j}^{s_{i j}^{k q}, h}\right)\left(x_{i j}\left(S_{i j}^{k q}, h\right)-x_{i j}(k, q)-x_{i j}(k, q)\right) \geq 0 \\
q=1,2,3, k=1, \cdots, n_{q}, j=1,2, i=1,2,3 \\
\left(1-\beta_{3}^{k+1, q}\right)\left(x_{i, 3}(k+1, q)-x_{i, 3}(k, q)-y_{i, 3}(k, q)\right) \geq 0 \\
q=1,2,3, k=1, \cdots, n_{q}-1, i=1,2,3
\end{gathered}
$$

Assuming that the current processing is for the $k$ th charge of the $q$ th cast on the $i$ th machine in the $j$ th group, then we define $\left.t_{i j}^{*}(k, q)\right)$ as its starting time on the $i$ th machine in the $j$ th group, and $t_{i j}^{* *}(k, q)$ as the corresponding end time.

The starting time and the processing time of charge which is being processed will not be changed.

$$
\begin{gathered}
\beta_{j}^{k q}\left(x_{i j}(k, q)-t_{i j}^{*}(k, q)\right)=0 \\
q=1,2,3, k=1, \cdots, n_{q}, j=1,2,3, i=1,2,3 \\
\beta_{j}^{k q}\left(y_{i j}(k, q)-\left(t_{i j}^{* *}(k, q)-t_{i j}^{*}(k, q)\right)\right)=0 \\
q=1,2,3, k=1, \cdots, n_{q}, j=1,2,3, i=1,2,3
\end{gathered}
$$

The starting time of charges which are not processed must be later than the production schedule time $T^{*}$.

$\left(1-\beta_{j}^{k q}\right)\left(x_{i j}(k, q)-T^{*}\right)>0 \quad q=1,2,3, k=1, \cdots, n_{q}, j=1,2,3, i=1,2,3(21)$

In the dynamic scheduling, the processing time of charges must be greater than or equal to the minimal processing time of charge, and also must be less than or equal to the maximum processing time of charge. This means that

$$
\begin{gathered}
y_{i j}(k, q)-\tau_{i, j, a_{k q}}^{\min } \geq 0 \quad q=1,2,3, k=1, \cdots, n_{q}, j=1,2,3, k=1,2,3 \\
y_{i j}(k, q)-\tau_{i, j, a_{k q}}^{\max } \leq 0 \quad q=1,2,3, k=1, \cdots, n_{q}, j=1,2,3, i=1,2,3
\end{gathered}
$$

where $\tau_{i, j, a_{k q}}^{\min }$ represents the minimal processing time of the $k$ th charge of the $q$ th cast on the $i$ th machine in the $j$ th group, and $\tau_{i, j, a_{k q}}^{\max }$ as the corresponding maximum processing time. $a_{k q}$ represents the steel grade that is being made.

The processing time of charge which has been processed will not be changed. The following constraint needs to be met.

$$
\begin{gathered}
\chi_{j}^{k q}\left(y_{i j}(k, q)-\left(t_{i j}^{* *}(k, q)-t_{i j}^{*}(k, q)\right)\right)=0 \\
q=1,2,3, k=1, \cdots, n_{q}, j=1,2,3, i=1,2,3
\end{gathered}
$$

where $\chi_{j}^{k q}$ represents the processing state of the $k$ th charge of the $q$ th cast of the $j$ th group. It is set to ' 1 ' if the charge has been processed. Otherwise it is set to ' 0 '.

\section{DYNAMIC OPTIMAL SCHEDULING MODEL FOR SMCC PRODUCTION PROCESS}

MONPM (M1) is established which considers changes in production mode, production schedule of charge, the interval characteristics of processing time based on the above analysis The purpose is to solve the following multi-objective optimization

$$
M \text { in } J^{1}=\left[J_{1}, J_{2}, J_{3}, J_{4}, J_{5}, J_{6}, J_{7}, J_{8}, J_{9}, J_{10}, J_{11}, J_{12}, J_{13}, J_{14}\right]^{T}
$$

where (15) (24) should be satisfied together with

$$
x_{i j}(k, q) \geq 0 \quad q=1,2,3, k=1, \cdots, n_{q}, j=1,2,3, i=1,2,3
$$

\section{DYNAMIC OPTIMAL SCHEDULING STRATEGY FOR SMCC PRODUCTION PROCESS}

There is still not very fast and effective method for solving the above model. This paper is not the blind pursuit of the optimal solution of nonlinear programming problem, but is to obtain near optimal solution in a reasonable time. A twostage dynamic optimal scheduling method is proposed including PPP and PTS as shown in Fig. 2, where $v_{m}$ is converter fault disturbance. The $\delta_{i 1}^{*}$ represents the starting time of $M_{i 1}$ which breaks down, and $\delta_{i 1}^{* *}$ is the corresponding end time.

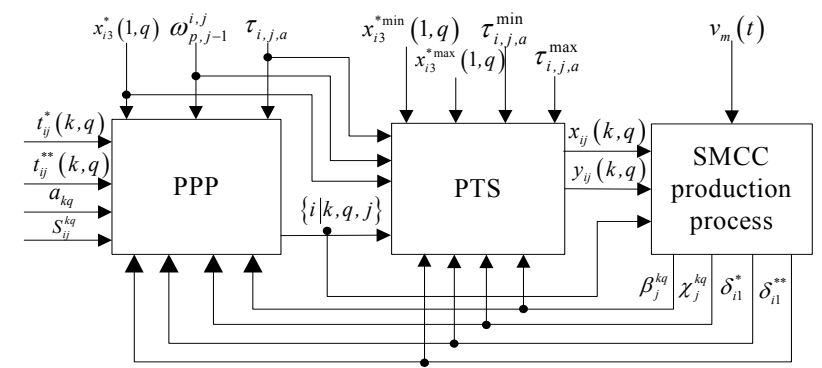

Fig. 2. Dynamic scheduling strategy 


\section{DYNAMIC OPTIMAL SCHEDULING METHOD FOR SMCC PRODUCTION PROCESS}

\subsection{Production Path Planning of Charges (PPP)}

PPP selects the processing machine for the $k$ th charge of the $q$ th cast in the $j$ th group type $(j=1,2)$ and determines the processing order of charges on machines. $L_{k q}$ must be arranged in the idle time of machine because of machine capacity. In the paper, the equipment availability for each machine is introduced, which means that the idle time of machine is expressed as $a v t_{i j}$ of $M_{i j}$.

The $a v t_{i j}$ is initialized as follows if $M_{i j}$ does not break down:

$a v t_{i j}=\max _{q=1,2,3}\left\{t_{i j}^{* *}\left(n_{q}, q\right)\right\}+\Delta t-\max \left\{x_{i 3}^{*}(1, q), \max _{\substack{q=1,2,3 \\ k=1, \cdots, n_{q}}}\left(\beta_{j}^{k q} t_{i j}^{* *}(k, q)\right)\right\}$

where $\Delta t$ is the pre-setting value. The $a v t_{i j}$ is initialized as follows if $M_{i j}$ breaks down:

$a v t_{i j}=\max _{q=1,2,3}\left\{t_{i j}^{* *}\left(n_{q}, q\right)\right\}+\Delta t-\max \left\{x_{i 3}^{*}(1, q), \max _{\substack{q=1,2,3 \\ k=1, \cdots, n_{q}}}\left(\beta_{j}^{k q} t_{i j}^{* *}(k, q)\right)\right\}-\left(\delta_{i 1}^{* *}-\delta_{i 1}^{*}\right)$

When $L_{k q}$ is arranged on the machine $M_{i j}$, the $a v t_{i j}$ should be updated as follows:

$$
a v t_{i j}=a v t_{i j}-\tau_{i, j, a_{k q}}
$$

The greater the value of $a v t_{i j}$ is, the longer idle time of $M_{i j}$ is. The $a v t_{i j}$ is closely related to the equipment load. $L_{k q}$ is arranged on the machine $M_{i j}$ whose $a v t_{i j}$ is greatest when PPP.

PPP firstly represents the charges as $\left\{L_{k q} \mid q=1,2,3, k=1, \cdots, n_{q}\right\}$, which has not been processed at continuous casting stage. Secondly, PPP selects the charges represented as $\left\{L_{k q}^{1}\right\}$ from $\left\{L_{k q}\right\}$ to PPP which are being processed or have been processed at steelmaking stage but are still not processed at RH refining stage. Finally, the charges represented as $\left\{L_{k q}^{2}\right\}$ are selected to PPP which are still not processed at steelmaking stage. All charges in $\left\{L_{k q}^{2}\right\}$ are firstly arranged on machines at refining stage and then are arranged on machines at steelmaking stage. In the PPP algorithm, the $b_{i j}^{k q}$ is defined as the processing unit of the $k$ th charge of the $q$ th cast on the $i$ th machine in the $j$ th group. The $z_{i j}^{*}(k, q)$ represents the initial starting time of the $k$ th charge of the $q$ th cast on the $i$ th machine in the $j$ th group, and $z_{i j}^{* *}(k, q)$ as the corresponding end time. The PPP algorithm is described as follows:

Step1: Select the charges $\left\{L_{k q} \mid q=1,2,3, k=1, \cdots, n_{q}\right\}$ from initial scheduling plan, which has not been processed at continuous casting stage, and initialize $\beta_{j}^{k q}, \chi_{j}^{k q}$ and $a v t_{i j}$.

Step2: Select the charges $\left\{L_{k q}^{1}\right\}$ from $\left\{L_{k q}\right\}$ to PPP which are been processing or have been processed at steelmaking stage but are still not processed at RH refining stage.
Step3: Select the charges $\left\{L_{k q}^{2}\right\}$ from $\left\{L_{k q}\right\}$ to PPP which are still not processed at steelmaking stage.

Step4: Calculate the starting time $z_{i, 3}^{*}(k, q)$ and the end time $z_{i, 3}^{* *}(k, q)$ of all units in the $\left\{L_{i j}^{2}\right\}$ at continuous casting stage.

Step5: Calculate the starting time $z_{p, 2}^{*}(k, q)$ and the end time $z_{p, 2}^{* *}(k, q)$ of all units in the $\left\{L_{k q}^{2}\right\}$ at $\mathrm{RH}$ refining stage. All units in $\left\{L_{k q}^{2}\right\}$ are reversely sorted by $z_{p, 2}^{*}(k, q)$, and then is expressed as $I_{3}$.

Step6: Select machine for unit $b_{p, 2}^{k, q}$ one by one in $I_{3}$. Select the $M_{p, 2}$ whose $a v t_{p, 2}$ is the greatest for unit $b_{p, 2}^{k, q}$. If there is only one machine whose $a v t_{p, 2}$ is the greatest, then the unit $b_{p, 2}^{k, q}$ is arranged on $M_{p, 2}$. If there are more than one machine whose $a v t_{p, 2}$ is the greatest, then select the one according to the minimal transportation time.

Step7: Recalculate the $z_{p, 2}^{*}(k, q)$ and $z_{p, 2}^{* *}(k, q)$ of unit $b_{p, 2}^{k, q}$ for selected the $p$ th machine. If the $L_{k q}$ is still the first unit in $I_{3}$, then select the $p$ th machine for $L_{k q}$ and Update the $a v t_{p, 2}$ of $M_{p, 2}$ according to the formula (29), else go to Step6.

Step8: Arrange all units in the $I_{3}$.

Step9: Calculate the starting time $z_{i, 1}^{*}(k, q)$ and the end time $z_{i, 1}^{* *}(k, q)$ of all units in $\left\{L_{k q}^{2}\right\}$ at steelmaking stage according to $z_{p, 2}^{*}(k, q)$ at $\mathrm{RH}$ refining stage and $\tau_{i, 1, a_{k q}}$. All units in $\left\{L_{k q}^{2}\right\}$ are reversely sorted by $z_{i, 1}^{*}(k, q)$ and expressed as $I_{4}$.

Step10: Arrange all units in the $I_{4}$ using above method.

\subsection{PTS}

After PPP, the processing machine is selected for the $k$ th charge of the $q$ th cast in the $j$ th group. The final starting time and processing time should be still determined using PTS.

Complexity of model and the difficulty of solving problem are greatly reduced because variable $i$ and $p$ have been determined through PPP. The weighted sum method is used to solve model, where it is supposed that

$$
\begin{aligned}
& Q_{i, 3}(1, q)=\left|x_{i, 3}(1, q)-x_{i, 3}^{*}(1, q)\right|+\left(x_{i, 3}(1, q)-x_{i, 3}^{*}(1, q)\right) \quad q=1,2,3(30) \\
& R_{i, 3}(1, q)=\left|x_{i, 3}(1, q)-x_{i, 3}^{*}(1, q)\right|-\left(x_{i, 3}(1, q)-x_{i, 3}^{*}(1, q)\right) \quad q=1,2,3(31)
\end{aligned}
$$

where $Q_{i, 3}(1, q), R_{i, 3}(1, q) \geq 0, Q_{i, 3}(1, q) \cdot R_{i, 3}(1, q)=0$, then

$$
\begin{aligned}
& \left(Q_{i, 3}(1, q)+R_{i, 3}(1, q)\right) / 2=\left|x_{i, 3}(1, q)-x_{i, 3}^{*}(1, q)\right| q=1,2,3 \\
& \left(Q_{i, 3}(1, q)-R_{i, 3}(1, q)\right) / 2=\left(x_{i, 3}(1, q)-x_{i, 3}^{*}(1, q)\right) \quad q=1,2,3 \\
& x_{i, 3}(1, q)=\left(Q_{i, 3}(1, q)-R_{i, 3}(1, q)\right) / 2+x_{i, 3}^{*}(1, q) \quad q=1,2,3
\end{aligned}
$$

Then model is converted to the line programming problem as follows. 


$$
\begin{aligned}
M \operatorname{in} J^{3}= & \sum_{q=1}^{3} C l_{q}\left(1-\beta_{3}^{1, q}\right)\left(Q_{i, 3}(1, q)+R_{i, 3}(1, q)\right) / 2+ \\
& \sum_{q=1}^{3} C 2_{q}\left(1-\beta_{3}^{2, q}\right)\left(x_{i, 3}(2, q)-\left(Q_{i, 3}(1, q)-R_{i, 3}(1, q)\right) / 2-x_{i, 3}^{*}(1, q)\right. \\
& \left.-y_{i, 3}(1, q)\right)+\sum_{q=1}^{3} \sum_{k=2}^{n_{q}-1} C 2_{i}\left(1-\beta_{3}^{k+1, q}\right)\left(x_{i, 3}(k+1, q)-x_{i, 3}(k, q)-\right. \\
& \left.y_{i, 3}(k, q)\right)+\sum_{q=1}^{3} C 3_{1 q}\left(1-\beta_{2}^{1, q}\right)\left(x_{i, 2}(1, q)-x_{p, 1}(1, q)-y_{p, 1}(1, q)\right. \\
& \left.-\omega_{p, 1}^{, 2}\right)+\sum_{q=1}^{3} C 3_{1 q}\left(1-\beta_{3}^{1, q}\right)\left(\left(Q_{i, 3}(1, q)-R_{i, 3}(1, q)\right) / 2+x_{i, 3}^{*}(1, q)-\right. \\
& \left.x_{p, 2}(1, q)-y_{p, 2}(1, q)-\omega_{p, 2}^{, 3}\right)+\sum_{q=1}^{3} \sum_{k=2}^{n_{q}} \sum_{j=1}^{2} C 3_{k q}\left(1-\beta_{j+1}^{k q}\right) \\
& \left(x_{i, j+1}(k, q)-x_{p, j}(k, q)-y_{p, j}(k, q)-\omega_{p, j}^{, j, j}\right)+(1-\alpha) \sum_{i=1}^{3} \sum_{j=1}^{n_{i}} C 4_{i}(1- \\
& \left.\beta_{1}^{s_{1,1}, h}\right)\left(x_{i, 1}\left(S_{i, 1}^{k,}, h\right)-x_{i, 1}(k, q)-y_{i, 1}(k, q)\right)+\sum_{q=1}^{3} C 5_{q}\left(x_{i, 3}\left(n_{q}, q\right)+\right. \\
& \left.y_{i, 3}\left(n_{q}, q\right)-T^{*}\right)
\end{aligned}
$$

S.T.

$$
\begin{aligned}
& \left(1-\beta_{2}^{1, q}\right)\left(x_{i, 2}(1, q)-x_{p, 1}(1, q)-y_{p, 1}(1, q)-\omega_{p, 1}^{i, 2}\right) \geq 0 \quad q=1,2,3(36) \\
& \left(1-\beta_{3}^{1, q}\right)\left(\left(Q_{i, 3}(1, q)-R_{i, 3}(1, q)\right) / 2+x_{i, 3}^{*}(1, q)-x_{p, 2}(1, q)-y_{p, 2}(1, q)-\omega_{p, 2}^{j, 3}\right) \geq 0 \\
& q=1,2,3 \\
& \left(1-\beta_{j+1}^{k q}\right)\left(x_{i, j+1}(k, q)-x_{p, j}(k, q)-y_{p, j}(k, q)-\omega_{p, j}^{i, j+1}\right) \geq 0 \\
& q=1,2,3, k=2, \cdots, n_{q}, j=1,2 \\
& \left(1-\beta_{j}^{S_{i j}^{k q}, h}\right)\left(x_{i j}\left(S_{i j}^{k q}, h\right)-x_{i j}(k, q)-y_{i j}(k, q)\right) \geq 0 \\
& q=1,2,3, k=1, \cdots, n_{q}, j=1,2 \\
& \left(1-\beta_{3}^{2, q}\right)\left(x_{i, 3}(2, q)-\left(Q_{i, 3}(1, q)-R_{i, 3}(1, q)\right) / 2-x_{i, 3}^{*}(1, q)-y_{i, 3}(1, q)\right) \geq 0 \\
& q=1,2,3 \\
& \left(1-\beta_{3}^{k+1, q}\right)\left(x_{i, 3}(k+1, q)-x_{i, 3}(k, q)-y_{i, 3}(k, q)\right) \geq 0 \\
& q=1,2,3, k=2, \cdots, n_{q}-1 \\
& \beta_{j}^{k q}\left(x_{i j}(k, q)-t_{i j}^{*}(k, q)\right)=0 \quad q=1,2,3, k=1, \cdots, n_{q}, j=1,2 \\
& \beta_{3}^{1, q}\left(\left(Q_{i, 3}(1, q)-R_{i, 3}(1, q)\right) / 2+x_{i, 3}^{*}(1, q)-t_{i j}^{*}(k, q)\right)=0 \quad q=1,2,3 \\
& \beta_{3}^{k q}\left(x_{i, 3}(k, q)-t_{i, 3}^{*}(k, q)\right)=0 \quad q=1,2,3, k=2, \cdots, n_{q} \\
& \beta_{j}^{k q}\left(y_{i j}(k, q)-\left(t_{i j}^{* *}(k, q)-t_{i j}^{*}(k, q)\right)\right)=0 \\
& q=1,2,3, k=1, \cdots, n_{q}, j=1,2,3 \\
& \left(1-\beta_{j}^{k q}\right)\left(x_{i j}(k, q)-T^{*}\right)>0 \quad q=1,2,3, k=1, \cdots, n_{q}, j=1,2 \\
& \left(1-\beta_{3}^{1, q}\right)\left(\left(Q_{i, 3}(1, q)-R_{i, 3}(1, q)\right) / 2+x_{i, 3}^{*}(1, q)-T^{*}\right)>0 \quad q=1,2,3(47) \\
& \left(1-\beta_{3}^{k q}\right)\left(x_{i, 3}(k, q)-T^{*}\right)>0 \quad q=1,2,3, k=2, \cdots, n_{q} \\
& y_{i j}(k, q)-\tau_{i, j, a_{k q}}^{\min } \geq 0 \quad q=1,2,3, k=1, \cdots, n_{q}, j=1,2,3 \\
& y_{i j}(k, q)-\tau_{i, j, a_{k q}}^{\max } \leq 0 \quad q=1,2,3, k=1, \cdots, n_{q}, j=1,2,3 \\
& \chi_{j}^{k q}\left(y_{i j}(k, q)-\left(t_{i j}^{* *}(k, q)-t_{i j}^{*}(k, q)\right)\right)=0 \\
& q=1,2,3, k=1, \cdots, n_{q}, j=1,2,3 \\
& x_{i j}(k, q) \geq 0 \quad q=1,2,3, k=1, \cdots, n_{q}, j=1,2 \\
& \left(Q_{i, 3}(1, q)-R_{i, 3}(1, q)\right) / 2+x_{i, 3}^{*}(1, q) \geq 0 \quad q=1,2,3 \\
& x_{i, 3}(k, q) \geq 0 \quad q=1,2,3, k=2, \cdots, n_{q}
\end{aligned}
$$

$$
\begin{array}{ll}
Q_{i, 3}(1, q) \geq 0 & q=1,2,3 \\
R_{i, 3}(1, q) \geq 0 & q=1,2,3
\end{array}
$$

where $C 1_{q}$ is a coefficient of penalty cost for the $q$ th cast not starting on time. $C 2_{q}$ is a coefficient of cast break loss penalty for the $q$ th cast. $C 3_{q j}$ is a coefficient of penalty cost for the waiting time; $C 4_{i}$ is coefficient of penalty cost for charges being discontinuous processed on $i$ th converter. $C 5_{q}$ is coefficient of penalty cost for maximum completion time.

\section{INDUSTRIAL APPLICATION}

\subsection{Application Background}

The Baosteel factory of China produces 1000 kinds steel grade. The number of refining processing ranging is from 1 to 4 , and refining routes are more than 20 . There are three parallel converters of $300 \mathrm{t}$ at steel making stage (1LD, 2LD, $3 \mathrm{LD})$, four kinds of refining machines $(1 \mathrm{RH}, 2 \mathrm{RH}, 3 \mathrm{RH}$, 1CAS, 2CAS, 3CAS, KIP, LF) at the refining stage, and three continuous casters $(1 \mathrm{CC}, 2 \mathrm{CC}, 3 \mathrm{CC})$. The practical scheduling system of SMCC production process only realizes information management, and the optimal dynamic scheduling isn't realized.

\subsection{Application example}

Taking production mode with three converters (1LD, 2LD, $3 \mathrm{LD})$ - three refining furnaces (1RH, 2RH, 3RH) - three casters $(1 \mathrm{CC}, 2 \mathrm{CC}, 3 \mathrm{CC})$ for example, the application of proposed method is explained in the following.

Initial scheduling plan is made by hybrid intelligent scheduling method (Yu, et al. [2006]) and includes 3 casts. The 3LD breaks down as shown in Fig. 3. It can be known that the production mode is two converters - three casters and is different from three converters - three casters mode before 3LD breaks down. Charges on 3LD must be transferred to another LD machines.

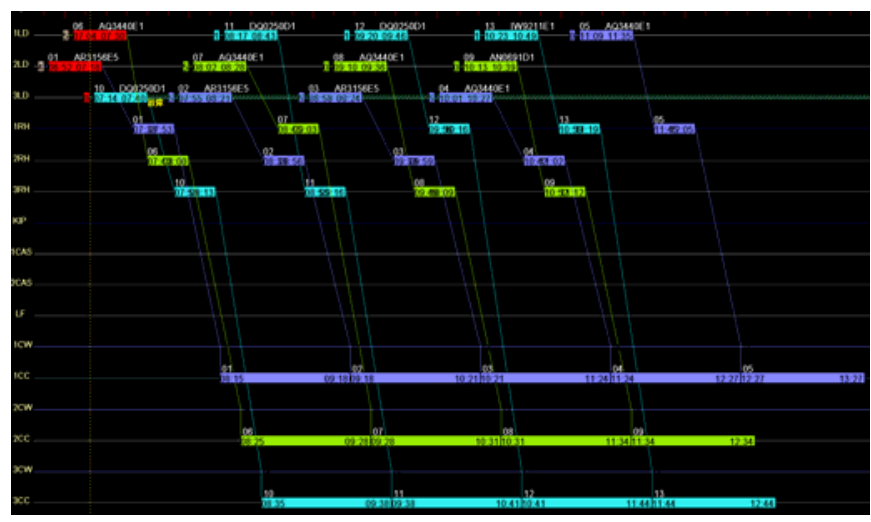

Fig. 3. Initial scheduling plan

Original scheduling optimization results are obtained which do not consider changes in production mode $(\alpha=1)$ and the interval characteristics of process time as shown in Fig. 4. It can be seen that the total length of idle time is 80 minutes in 
the steelmaking stage, indicating that equipment capacity in steelmaking stage is not fully utilized. The total length of redundant waiting time is 57 minutes and completion time of all charges is 392 minutes. Other new scheduling results are obtained through PTS which considers changes in production mode $(\alpha=0)$ and the interval characteristics of process time as shown in Fig. 5. It can be known that there is no idle time on converters. Equipment capacity in steelmaking stage is fully utilized and more molten steel can be produced. The total sum of redundant waiting time is 0 minutes and completion time of all charges is 305 minutes.

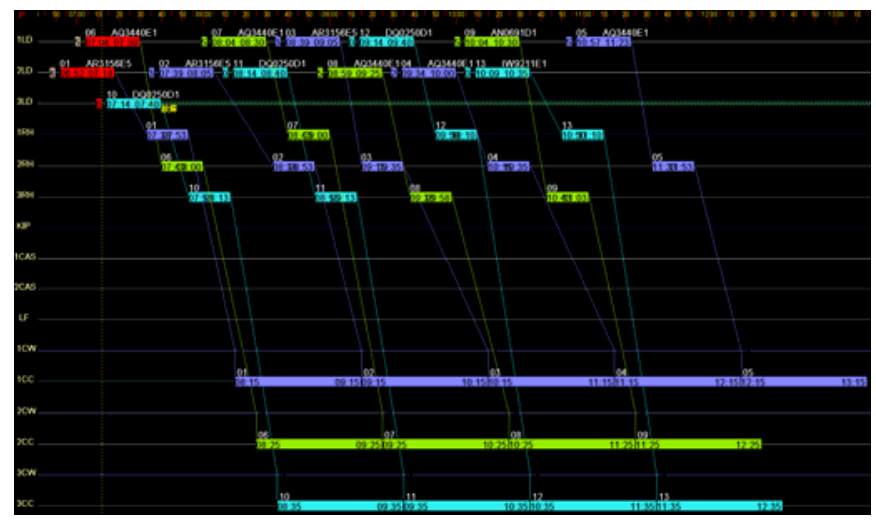

Fig. 4. Original scheduling optimization results

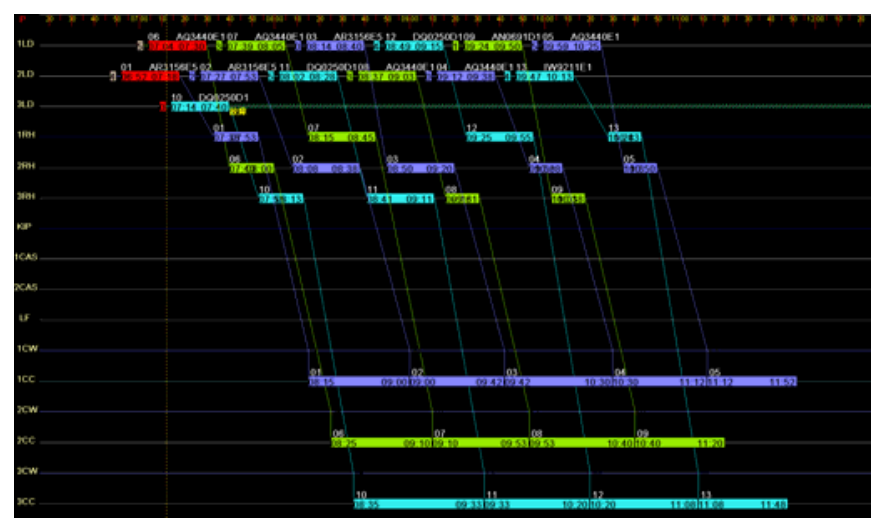

Fig. 5. New scheduling optimization results

\subsection{Application effect}

Using the proposed method, average daily load ratio of converters has been raised from $60.85 \%$ up to $67.12 \%$. The average daily load ratio of refining furnaces has been increased from $40.03 \%$ up to $45.40 \%$. The average daily load ratio has been improved from $50.44 \%$ up to $56.26 \%$. The redundant waiting time for molten steel reduces from 234 minutes to 166 minutes. As a result, the dynamic scheduling time is reduced from more than 1 minute to within 10 seconds.

\section{CONCLUSIONS}

Dynamic optimal scheduling method for SMCC is investigated in this paper. When the original scheduling plan is unfeasible because of converter fault, the new feasible scheduling plan is generated by dynamic scheduling. The
MONPM for dynamic scheduling is established which considers changes in production mode, production schedule of charge, the interval characteristics of process time. A twostage dynamic optimal scheduling method is proposed including PPP and PTS. DOSSS is developed based on above proposed method and is successfully applied to the scheduling of the largest iron and steel company (BaoSteel) in China. Applied results show that the proposed method can efficiently reduce scheduling time, significantly increase the outputs of converters and dramatically shorten the redundant waiting time for molten steel.

\section{REFERENCES}

Kumar V., Kumar S., Tiwari M. K., et al (2006). Auctionbased approach to resolve the scheduling problem in the steel making process. International Journal of Production Research, 44(8), 1503-1522.

Tang L. X., Liu J. Y., Rong A. Y., Yang Z. H. (2000). A mathematical programming model for scheduling steelmaking-continuous casting production. European Journal of Operational Research, 120(2), 423-435.

Tang L. X., Luh P. B., Liu J. Y., et al (2002). Steel-making process scheduling using Lagrangian relaxation. International Journal of Production Research, 40(1), 5570.

Xuan H., Tang L. X. (2007). Scheduling a hybrid flowshop with batch production at the last stage. Computers and Operations Research, 34(9), 2718-2733.

Harjunkoski I., Grossmann I. E. (2001). A decomposition approach for the scheduling of a steel plant production. Computers and Chemical Engineering, 25(11-12), 16471660.

Mohanty P. P. (2004). An Agent - oriented approach to resolve the production planning complexities for a modern steel manufacturing system. International Journal Advance Manufacturing Technology, 24(3-4), 199-205.

Cowling P. I., Ouelhadj D., Petrovic S. (2003). A multi Agent architecture for dynamic scheduling of steel hot rolling. Journal of Intelligent Manufacturing, 14(5), 457470.

Ouelhadj D., Petrovic S., Cowling P. I., et al (2004). Inter Agent cooperation and communication for Agent - based robust dynamic scheduling in steel production. Advanced Engineering Informatics, 18(3), 161-172.

Tang L. X., Guan J., Hu G. F. (2010). Steelmaking and refining coordinated scheduling problem with waiting time and transportation consideration. Computers \& Industrial Engineering, 58(2), 239-248.

Pacciarelli D., Pranzo M. (2004). Production scheduling in a steelmaking-continuous casting plant. Computers and Chemical Engineering, 28(12), 2832-2835.

Lu C. Y., Stankovic J. A., Son S. H., et al (2002). Feedback control real-time scheduling: framework, modeling, and algorithms. Real-Time Systems, 23(1-2), 85-126.

Yu S. P., Zheng B. L., Chai T. Y. (2006). Hybrid intelligent scheduling method and its application for steelmaking and continuous casting. Journal of East China University of Science and Technology(Natural Science Edition), 32(7), 844-848. 